\title{
Neurological update: MOG antibody disease
}

\author{
Ray Wynford-Thomas ${ }^{1,2} \cdot$ Anu Jacob $^{3} \cdot$ Valentina Tomassini $^{1,2,4}(\mathbb{0}$
}

Received: 17 August 2018 / Revised: 9 November 2018 / Accepted: 12 November 2018 / Published online: 19 December 2018 (c) The Author(s) 2018

\begin{abstract}
Myelin oligodendrocyte glycoprotein (MOG) antibody disease (MOG-AD) is now recognised as a nosological entity with specific clinical and paraclinical features to aid early diagnosis. Although no age group is exempt, median age of onset is within the fourth decade of life, with optic neuritis being the most frequent presenting phenotype. Disease course can be either monophasic or relapsing, with subsequent relapses most commonly involving the optic nerve. Residual disability develops in 50-80\% of patients, with transverse myelitis at onset being the most significant predictor of long-term outcome. Recent advances in MOG antibody testing offer improved sensitivity and specificity. To avoid misdiagnosis, MOG antibody testing should be undertaken in selected cases presenting clinical and paraclinical features that are felt to be in keeping with MOG-AD, using a validated cell-based assay. MRI characteristics can help in differentiating MOG-AD from other neuroinflammatory disorders, including multiple sclerosis and neuromyelitis optica. Cerebrospinal fluid oligoclonal bands are uncommon. Randomised control trials are limited, but observational open-label experience suggests a role for high-dose steroids and plasma exchange in the treatment of acute attacks, and for immunosuppressive therapies, such as steroids, oral immunosuppressants and rituximab as maintenance treatment.
\end{abstract}

Keywords Myelin oligodendrocyte glycoprotein (MOG) antibodies $\cdot$ Multiple sclerosis (MS) $\cdot$ Neuromyelitis optica $(\mathrm{NMO}) \cdot$ Optic neuritis $(\mathrm{ON}) \cdot$ Cerebrospinal fluid (CSF) · MRI

\section{Introduction}

Myelin oligodendrocyte glycoprotein (MOG) is a glycoprotein located on the myelin surface and found exclusively in the central nervous system (CNS) [1,2]. Although its exact role remains unclear, it is thought to act as a cellular adhesive molecule, to be involved as a regulator of oligodendrocyte microtubule stability and to mediate complement cascade [3].

Valentina Tomassini

tomassiniv@cardiff.ac.uk

1 Division of Psychological Medicine and Clinical Neurosciences, Cardiff University School of Medicine, University Hospital of Wales, Heath Park, Cardiff CF14 4XN, UK

2 Helen Durham Centre for Neuroinflammation, University Hospital of Wales, Cardiff, UK

3 Walton Centre NHS Foundation Trust, Liverpool, UK

4 Cardiff University Brain Research Imaging Centre (CUBRIC), School of Psychology, Cardiff, UK
MOG antibodies have been extensively studied over the last 30 years, with some early experimental studies hypothesising a pathogenic role in CNS inflammatory diseases [4, 5]. This hypothesis was later supported by their discovery in the sera and cerebrospinal fluid (CSF) of patients with multiple sclerosis (MS), using techniques such as enzyme-linked immunosorbent assay and Western blot [6-11]. However, the more recent development of highly sensitive and specific methods for MOG antibody detection using cell-based assays, along with new diagnostic classification of similar neuroinflammatory conditions, has made it possible to identify a subset of patients with antibodies to MOG who express a clinical phenotype distinct from MS or from neuromyelitis optica (NMO) [12-15]. Therefore, MOG antibody disease (MOG-AD) is now recognised as a distinct nosological entity with specific management and therapeutic requirements. In this article, we review the clinical features, investigations and challenges of managing patients with MOG-AD. 


\section{Clinical features of MOG antibody disease}

MOG-AD is an inflammatory demyelinating condition of the CNS characterised by a monophasic or relapsing course of neurological dysfunction, which does not meet the typical criteria for MS or other known neuroinflammatory conditions and occurs in the presence of serum MOG antibodies detected using specific cell-based assays [16].

Although in most cases demyelination associated with MOG antibodies occurs without any apparent inciting or predisposing event/illness, it has been associated with demyelinating $N$-methyl-D-aspartate receptor encephalitis, post-infectious demyelination following herpes simplex virus, Borrelia and Epstein-Barr virus infections and, more rarely, with typical relapsing MS [17-27]. Whether MOG antibodies play a pathogenic role in all these conditions, or if they represent a bystander effect or epiphenomenon, remains unclear [28].

MOG-AD can occur in all decades of life, with a slight predominance in women and with median age of onset in the early to mid-thirties [17, 18, 29-33]. The most common presenting feature is optic neuritis $(\mathrm{ON})$, occurring in $54-61 \%$ of patients, followed by myelitis, acute disseminated encephalomyelitis (ADEM) or an ADEM-like presentation (e.g., brainstem attack) [18, 29, 31, 32].

A relapsing course has been reported in $44-83 \%$ of patients $[18,29,32]$ and more commonly involves the optic nerve [30-32]. MOG-positive ON is frequently bilateral and associated with optic nerve head swelling $[34,35]$. The impact of relapses on disability is variable: some studies report no difference between monophasic and relapsing disease courses, [29] whilst others report worsening disability associated with higher relapse frequency [32]. In case-based series, residual disability develops in $50-80 \%$ of patients, $[18,31,32]$ with transverse myelitis at onset being the most significant predictor of long-term disability [32]. Several case-based series of seizures associated with MOG-AD have also been reported [36-38].

At presentation, MOG-positive patients are thought to be at lower risk of further relapses than aquaporin 4 (AQP4)positive patients [29] and have better visual and motor outcomes [29, 32]. In comparison to children with AQP4positive neuromyelitis optica spectrum disorder (NMOSD), those with MOG-AD tend to be younger, less likely to present with area postrema syndrome, but more likely to present with ADEM. In addition, they tend to have a longer time to relapse and lower disability at follow-up (2 years) [17]. Compared to AQP4-positive ON, MOG-positive patients have better visual field outcomes, [39] although recurrence of ON is significantly more frequent [40].

Table 1 summarises the clinical and paraclinical features of MOG-AD, as well as the characteristics that help in distinguishing MOG-AD from more common forms of neuroinflammation, i.e., MS and NMO.

\section{Paraclinical investigations for MOG antibody disease}

\section{Magnetic resonance imaging (MRI)}

Brain MRI scans are abnormal in approximately $45 \%$ of patients at onset [29,31], with percentages increasing later in the course of the disease (i.e., up to $77 \%$ of patients) [18]. The majority have bilateral lesions at onset $[29,30]$ and around one-third have sub-tentorial lesions [18], predominantly in the brainstem [29]. Typically, lesions are few (three or less) and appear as "fluffy", i.e., poorly demarcated hyperintensities on T2-weighted images. Dawson's fingers, U- or $\mathrm{S}$-shaped lesions and ovoid lesions adjacent to the body of lateral ventricles are found less commonly [29, 46]. When compared to MS-associated ON or AQP4-positive NMOSDassociated ON, the MRI appearance of the optic nerve in MOG-associated ON is more oedematous and shows extensive inflammatory lesions, usually sparing chiasm and optic tracts [35]. Thalamic and pontine lesions are more common in MOG-AD compared to AQP4-positive disease [29]. In children, bilateral thalamic lesions at onset are frequent and can be found in about $60 \%$ of patients [30]. Compared to AQP4-positive patients, cerebellar peduncle lesions are only found in MOG-positive children [17].

Just over half of MOG-AD patients have T2 hyperintense lesions in the spinal cord, with most lesions being short and predominantly occurring in the cervical or thoracic region [18]. In a study that explored MRI appearances in children, around 27\% had abnormal initial spinal cord MRI scans, two-third of which showed a longitudinally extensive transverse myelitis (LETM) [30].

\section{Cerebrospinal fluid}

CSF pleocytosis occurs in $44-85 \%$ of patients [18, 29, 31, $32]$ and is more common in children [30,31]. Positive oligoclonal bands (OCBs) are unusual, occurring in only $6-17 \%$, $[18,29-32]$ and CSF protein is raised in around a third of cases [31].

\section{Serum}

It is currently recommended that MOG antibody immunoglobulin $\mathrm{G}(\mathrm{IgG})$ is detected in serum, using a cell-based assay (indirect fluorescence test or fluorescence-activated cell sorting) and employing full length human MOG as the target antigen [12, 13, 47]. As false positive MOG antibody results can occur, testing for MOG antibodies should 


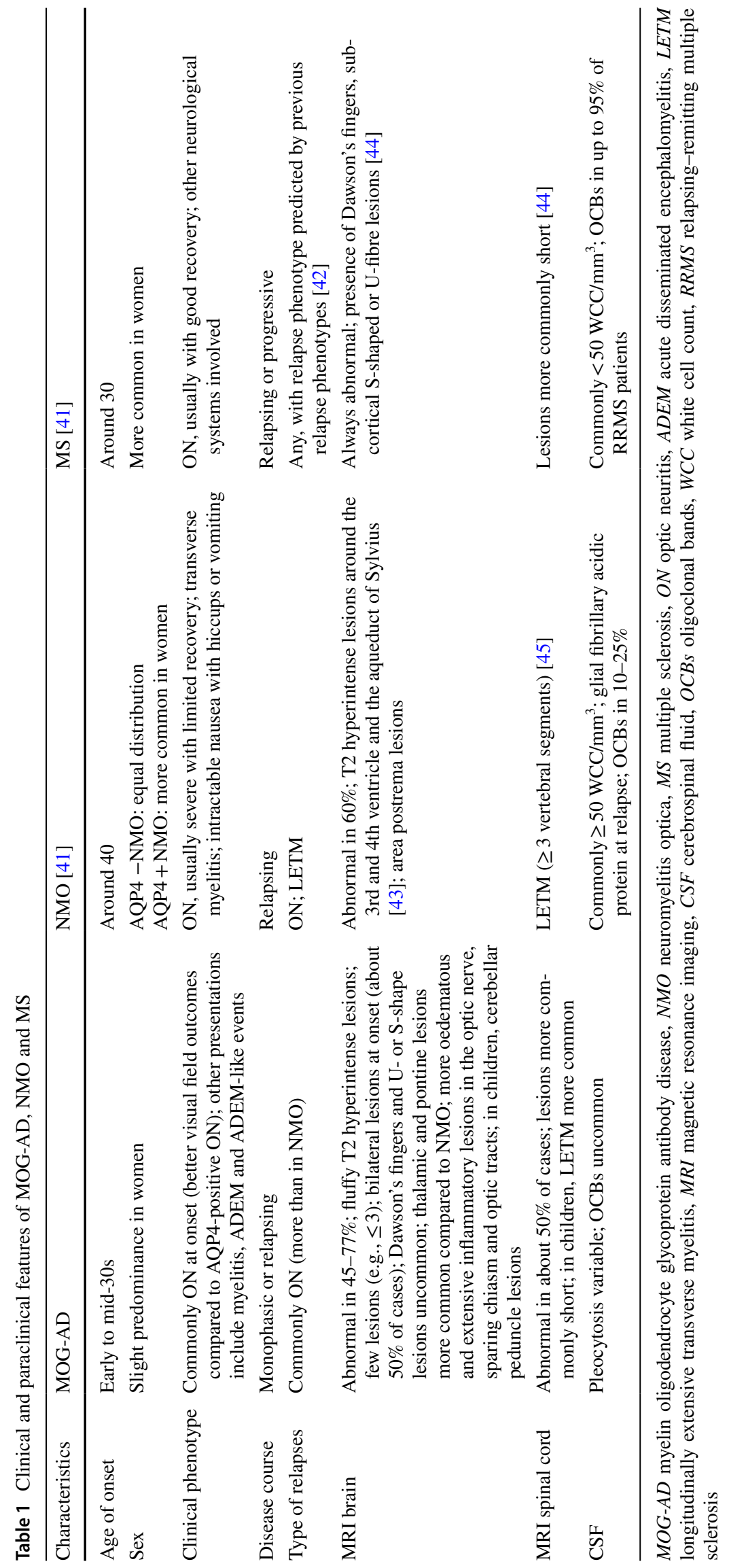


be restricted to selected cases only, whereby the clinical and paraclinical features are felt to be in keeping with MOG-AD [16].

MOG antibody titres have been found to be higher in relapse than in remission [29]. A decreasing titre is usually found in a monophasic disease course and conversion to antibody negativity, which has been shown to occur within around 8-36 months from an acute event, has been associated with no further relapses [18, 32]. However, following a negative result, antibodies can become positive again, even after a few years [32, 47]. In children, persisting high-titre MOG antibodies (e.g., over 24 months) are associated with a risk of relapse [20].

There is no definite consensus regarding regular antibody monitoring. However, as antibodies can increase in relapse and can subsequently become negative, it could be argued that there is a role for regular monitoring at diagnosis, as well as throughout the course of MOG-AD, when reduced antibody levels may indicate disease remission. Some have suggested that a re-test interval of 6-12 months may be helpful [16]. Table 2 summarises when MOG antibody testing is indicated.

\section{Biopsy}

In brain lesions of patients with positive MOG antibodies and the suspicion of MOG-AD, neuropathology seems compatible with pattern II histopathology of MS, i.e., with lesions presenting complement and IgG deposits at the sites of ongoing demyelination [22, 48].

\section{MOG antibody positivity and the diagnosis of MOG-AD: "red flags"}

The 2018 International Recommendations have highlighted "red flags" that should prompt a physician to doubt the relevance of a positive result [16]. In these cases, re-testing is advised, preferably using a different cell-based assay, and expert-opinion should be sought. "Red flags" include progressive disease; sudden onset of symptoms or continuous worsening of symptoms over weeks; combined central and peripheral demyelination, as MOG is not expressed in the peripheral nervous system; a MRI lesion adjacent to the lateral ventricle that is ovoid/round or associated with an inferior temporal lobe lesion, or Dawson's finger-type lesions; an active brain MRI over time with silent increase in lesion burden between relapses; bi- or tri-specific measles, rubella and zoster virus reaction in the CSF; serum MOG-IgG levels at or just barely above the assay-specific cut-off, especially if the clinical picture is atypical; positive MOG-IgM and/or MOG-IgA result with negative MOG-IgG; MOG-IgG positivity in the CSF, but not in the serum, given that MOG-IgG is typically produced extra-thecally; AQP4-IgG/MOG-IgG "double-positive" test results, which are very rare and should prompt re-testing for both antibodies [16]. In all these cases, caution should be exerted in diagnosing MOG-AD and clinical and paraclinical follow-up is advised.

\section{Pharmacological management of MOG antibody disease}

Thus far, there are no controlled treatment trials in MOG-AD and observational, open-label experience is limited. Therefore, care must be taken when interpreting or extrapolating results from studies or reports. Due to these limitations, current treatment protocols for MOG-AD tend to follow those of AQP4- NMO.

\section{Acute treatment}

Intravenous methylprednisolone (IVMP) for the treatment of MOG antibody positive $\mathrm{ON}$ and/or myelitis (dose ranging from 1 to $2 \mathrm{~g}$ once a day for 3-5 days) has been reported to be effective in some cases, with partial or no recovery in $50 \%$. Even if treatment with IVMP is effective initially, further courses may not be [33].

Plasma exchange (regime varying from three to five cycles) is more commonly used as a second-line treatment following steroid resistance and can result in around $40 \%$ of cases of MOG antibody $\mathrm{ON}$ and/or myelitis having a complete or almost complete recovery [33].

Table 2 When to test for MOG antibody

\begin{tabular}{ll}
\hline Test if & Clinical/paraclinical features are suggestive of MOG-AD (2018 International Recommendations) [16] \\
& Diagnosis of MS is made, interferon beta or natalizumab has been started, but efficacy is unexpect- \\
& edly poor and clinical/paraclinical features are compatible with MOG-AD \\
& MOG antibody positive, but clinical/paraclinical features not suggestive of MOG-AD (see "MOG \\
antibody positivity and the diagnosis of MOG-AD: "red flags"') \\
Clinical/paraclinical features continue to be suggestive of MOG-AD, but MOG antibody is negative \\
Likelihood of further events is sought, following MOG-AD diagnosis
\end{tabular}

$M O G$ myelin oligodendrocyte glycoprotein, $M O G-A D$ myelin oligodendrocyte glycoprotein antibody disease 


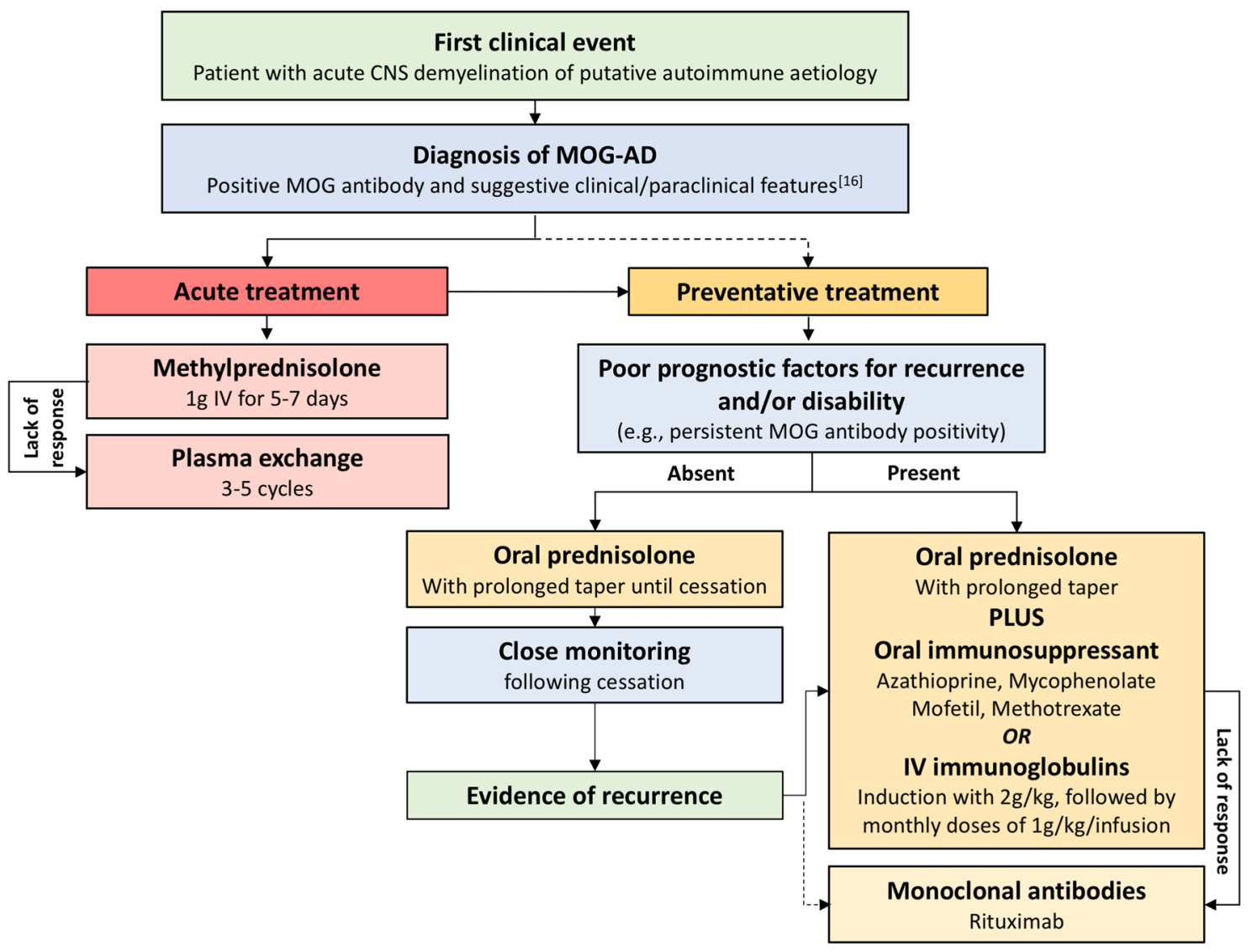

Fig. 1 Management of MOG-AD. This proposed therapeutic management of MOG-AD is based on current evidence. Acute treatment with steroids and, if needed, subsequent plasma exchange are advised as soon as possible after an acute event. After diagnosis and, usually, acute treatment, initiation of disease-modifying therapy is advised. The choice of the disease-modifying agent should be guided by the presence or absence of poor prognostic factors for recurrence and/ or disability. On this basis, treatment may require a prolonged taper with oral steroids (advised in the absence of poor prognostic factors) or the use of oral immunosuppressants or intravenous immunoglobu-

\section{Disease-modifying treatment}

Long-term treatments with prednisolone (dose $>10 \mathrm{mg} /$ day for patients $>40 \mathrm{~kg}$ in weight and $>5 \mathrm{mg}$ for patients $\leq 40 \mathrm{~kg}$ in weight [31]), intravenous immunoglobulins (induction course dose of $2 \mathrm{~g} / \mathrm{kg}$ with subsequent monthly doses of $1 \mathrm{~g} / \mathrm{kg} /$ infusion [31]), rituximab, mycophenolate mofetil, methotrexate or azathioprine have all been reported to reduce annualised relapse rate in MOG-AD [31, 33, 37]. However, treatment with rituximab can be associated with new attacks within the few weeks following the first infusion, possibly due to a temporary increase in B-cell activating factor and autoantibody levels, as also observed in some AQP4-positive NMO patients [33]. Although numbers are small, treatment with lins along with oral steroids (advised as first choice in the presence of poor prognostic factors). If a lack of response to immunosuppressants is demonstrated or a disabling recurrence of the disease occurs after cessation of oral steroids, it is appropriate to consider monoclonal antibodies. While it is reasonable and common practice to treat relapsing patients with long-term immunosuppression, the duration of disease-modifying treatment remains uncertain. $C N S$ central nervous system, $M O G-A D$ myelin oligodendrocyte glycoprotein antibody disease, $M O G$ myelin oligodendrocyte glycoprotein, $I V$ intravenous

natalizumab has been shown to be ineffective in preventing relapses, whereas treatment with ofatumumab was able to reduce annualised relapse rate from 2.1 to 0.66 [33]. One study found that the rate of treatment failure was lower in patients on maintenance steroids $(5 \%$; median treatment duration of 10 months) compared to non-steroidal maintenance therapies (38\%) [31].

The role of oral steroids as adjunctive treatment with immunosuppressants remains unclear. A study found that patients with MOG antibody ON and/or myelitis treated with azathioprine, but not adjunctively with oral steroids, experienced relapses more commonly than patients who underwent combination therapy [33]. Furthermore, relapses seem to occur more frequently when oral prednisolone doses are 
dropped below $10 \mathrm{mg} /$ day or early after steroid cessation [31, 32].

Treatment with interferon beta has been shown to be ineffective and can increase disease activity [33,37]. Similarly, glatiramer acetate has been shown to be ineffective when administered in children [37].

Figure 1 summarises the proposed management of MOG$\mathrm{AD}$, based on current evidence.

\section{Conclusions}

The diagnosis of MOG-AD is crucial to plan appropriate management. Suggestive clinical and paraclinical features, in association with MOG antibody positivity, aid the identification of MOG-AD from other inflammatory demyelinating diseases, but, in many cases, a definitive diagnosis remains difficult. Therefore, the definition of MOG-AD-specific diagnostic criteria, as well as the identification of markers of disease status, remains a goal of research. Once the disease has been diagnosed, uncertainty remains over the best treatment approach and clinical trials for the pharmacological management of MOG-AD are still needed.

\section{Compliance with ethical standards}

Conflicts of interest Dr. R. Wynford-Thomas' clinical research fellow Grant is co-funded by Novartis. Dr. A. Jacob has received research Grants from Biogen Idec, Alexion Pharmaceuticals and speaker fees from Biogen, Chugai, Sanofi-Genzyme and Terumo-BC. Dr. V. Tomassini has received research support and honoraria from Biogen Idec, and honoraria and travel Grants from Biogen Idec and Novartis.

Open Access This article is distributed under the terms of the Creative Commons Attribution 4.0 International License (http://creativeco mmons.org/licenses/by/4.0/), which permits unrestricted use, distribution, and reproduction in any medium, provided you give appropriate credit to the original author(s) and the source, provide a link to the Creative Commons license, and indicate if changes were made.

\section{References}

1. Schluesener $\mathrm{H}$ et al (1987) A monoclonal antibody against a myelin oligodendrocyte glycoprotein induces relapses and demyelination in central nervous system autoimmune disease. J Immunol 139(12):4016-4021

2. Bernard C et al (1997) Myelin oligodendrocyte glycoprotein: a novel candidate autoantigen in multiple sclerosis. J Mol Med 75(2):77-88

3. Johns T, Bernard C (1999) The structure and function of myelin oligodendrocyte glycoprotein. J Neurochem 72(1):1-9

4. Linington C, Lassmann H (1987) Antibody responses in chronic relapsing experimental allergic encephalomyelitis: correlation of serum demyelinating activity with antibody titre to the myelin/oligodendrocyte glycoprotein (MOG). J Neuroimmunol 17(1):61-69
5. Linington $\mathrm{C}$ et al (1988) Augmentation of demyelination in rat acute allergic encephalomyelitis by circulating mouse monoclonal antibodies directed against a myelin/oligodendrocyte glycoprotein. Am J Pathol 130(3):443-454

6. Karni A et al (1999) Elevated levels of antibody to myelin oligodendrocyte glycoprotein is not specific for patients with multiple sclerosis. Arch Neurol 56(3):311-315

7. Tomassini V et al (2007) Anti-myelin antibodies predict the clinical outcome after a first episode suggestive of MS. Mult Scler 13(9): 1086-1094

8. Berger T et al (2003) Antimyelin antibodies as a predictor of clinically definite multiple sclerosis after a first demyelinating event. N Engl J Med 349:139-145

9. Rauer S et al (2006) Antimyelin antibodies and the risk of relapse in patients with a primary demyelinating event. J Neurol Neurosurg Psychiatry 77:739-742

10. Pittock S et al (2007) Myelin oligodendrocyte glycoprotein antibodies in pathologically proven multiple sclerosis: frequency, stability and clinicopathologic correlations. Mult Scler 13(1):7-16

11. Kuhle $\mathbf{J}$ et al (2007) Lack of association between antimyelin antibodies and progression to multiple sclerosis. N Engl J Med 356(4):371-378

12. Tanaka M, Tanaka K (2014) Anti-MOG antibodies in adult patients with demyelinating disorders of the central nervous system. J Neuroimmunol 270(1-2):98-99

13. Waters $\mathrm{P}$ et al (2015) MOG cell-based assay detects non-MS patients with inflammatory neurologic disease. Neurol Neuroimmunol Neuroinflamm 2(3):e89

14. Wingerchuk D et al (2015) International consensus diagnostic criteria for neuromyelitis optica spectrum disorders. Neurology 85(2): 177-189

15. Thompson A et al (2018) Diagnosis of multiple sclerosis: 2017 revisions of the McDonald criteria. Lancet Neurol 17(2):162-173

16. Jarius $\mathrm{S}$ et al (2018) MOG encephalomyelitis: international recommendations on diagnosis and antibody testing. J Neuroinflamm $15: 134$

17. Hacohen $Y$ et al (2017) Diagnostic algorithm for relapsing acquired demyelinating syndromes in children. Neurology 89(3):269-278

18. Mariotto $\mathrm{S}$ et al (2017) Clinical spectrum and IgG subclass analysis of anti-myelin oligodendrocyte glycoprotein antibody-associated syndromes: a multicenter study. J Neurol 264(12):2420-2430

19. Kezuka T, Ishikawa H (2018) Diagnosis and treatment of antimyelin oligodendrocyte glycoprotein antibody positive optic neuritis. Jpn J Ophthalmol 62(2):101-108

20. Hennes E et al (2017) Prognostic relevance of MOG antibodies in children with an acquired demyelinating syndrome. Neurology 89:900-908

21. Wong Y et al (2018) Paediatric acute disseminated encephalomyelitis followed by optic neuritis: disease course, treatment response and outcome. Eur J Neurol 25(5):782-786

22. Spadaro $\mathrm{M}$ et al (2015) Histopathology and clinical course of MOG-antibody-associated encephalomyelitis. Ann Clin Transl Neurol 2(3):295-301

23. Zhou L et al (2017) Cerebral cortical encephalitis followed by recurrent CNS demyelination in a patient with concomitant antiMOG and anti-NMDA receptor antibodies. Mult Scler Relat Disord 18:90-92

24. Zhou J et al (2018) An unusual case of anti-MOG CNS demyelination with concomitant mild anti-NMDAR encephalitis. J Neuroimmunol. https://doi.org/10.1016/j.jneuroim.2018.03.019. (Epub ahead of print)

25. Nakamura M et al (2017) A case of MOG antibody-positive bilateral optic neuritis and meningoganglionitis following a genital herpes simplex virus infection. Mult Scler Relat Disord $17: 148-150$ 
26. Vieira J, Sequeira J, Brito M (2017) Postinfectious anti-myelin oligodendrocyte glycoprotein antibody positive optic neuritis and myelitis. J Child Neurol 32(12):996-999

27. Nakamura $Y$ et al (2017) Anti-MOG antibody-positive ADEM following infectious mononucleosis due to a primary EBV infection: a case report. BMC Neurol 17(1):76

28. Peschl $\mathrm{P}$ et al (2017) Human antibodies against the myelin oligodendrocyte glycoprotein can cause complement-dependent demyelination. J Neuroinflamm 14:208

29. Cobo-Calvo A et al (2018) Clinical spectrum and prognostic value of CNS MOG autoimmunity in adults: the MOGADOR study. Neurology. https://doi.org/10.1212/WNL.0000000000005560. (Epub ahead of print)

30. Cobo-Calvo A et al (2017) MOG antibody-related disorders: common features and uncommon presentations. J Neurol 264(9):1945-1955

31. Ramanathan $\mathrm{S}$ et al (2018) Clinical course, therapeutic responses and outcomes in relapsing MOG antibody-associated demyelination. J Neurol Neurosurg Psychiatry 89:127-137

32. Jurynczyk $\mathrm{M}$ et al (2017) Clinical presentation and prognosis in MOG-antibody disease: a UK study. Brain 140(12):3128-3138

33. Jarius $\mathrm{S}$ et al (2016) MOG-IgG in NMO and related disorders: a multicenter study of 50 patients. Part 2: epidemiology, clinical presentation, radiological and laboratory features, treatment responses, and long-term outcome. J Neuroinflamm 13:280

34. Ramanathan $\mathrm{S}$ et al (2015) Radiological differentiation of optic neuritis with myelin oligodendrocyte glycoprotein antibodies, aquaporin-4 antibodies, and multiple sclerosis. Mult Scler J 22(4):470-482

35. Biotti D et al (2017) Optic neuritis in patients with anti-MOG antibodies spectrum disorder: MRI and clinical features from a large multicentric cohort in France. J Neurol 264(10):2173-2175

36. Hamid S et al (2018) Seizures and encephalitis in myelin oligodendrocyte glycoprotein IgG disease vs aquaporin $4 \mathrm{IgG}$ disease. JAMA Neurol 75(1):65-71
37. Hacohen $\mathrm{Y}$ et al (2018) Disease course and treatment responses in children with relapsing myelin oligodendrocyte glycoprotein antibody-associated disease. JAMA Neurol 75(4):478-487

38. Ogawa $\mathrm{R}$ et al (2017) MOG antibody-positive, benign, unilateral, cerebral cortical encephalitis with epilepsy. Neurol Neuroimmunol Neuroinflamm 4(2):e322

39. Stiebel-Kalish $\mathrm{H}$ et al (2017) Retinal nerve fiber layer may be better preserved in MOG-IgG versus AQP4-IgG optic neuritis: a cohort study. PLoS One 12(1):e0170847

40. Pache F et al (2016) MOG-IgG in NMO and related disorders: a multicenter study of 50 patients. Part 4: afferent visual system damage after optic neuritis in MOG-IgG-seropositive versus AQP4-IgG-seropositive patients. J Neuroinflamm 13:282

41. Jurynczyk M et al (2015) Overlapping CNS inflammatory diseases: differentiating features of NMO and MS. J Neurol Neurosurg Psychiatry 86:20-25

42. Kalincik T et al (2014) Risk of relapse phenotype recurrence in multiple sclerosis. Mult Scler 20(11):1511-1522

43. Matthews L et al (2013) Distinction of seropositive NMO spectrum disorder and MS brain lesion distribution. Neurology 80(14):1330-1337

44. Tomassini V, Palace J (2009) Multiple sclerosis lesions: insights from imaging techniques. Expert Rev Neurother 9(9):1341-1359

45. Wingerchuk D et al (2007) The spectrum of neuromyelitis optica. Lancet Neurol 6(9):805-815

46. Jurynczyk M et al (2017) Distinct brain imaging characteristics of autoantibody-mediated CNS conditions and multiple sclerosis. Brain 140(3):617-627

47. Spadaro M et al (2016) Autoantibodies to MOG in a distinct subgroup of adult multiple sclerosis. Neurol Neuroimmunol Neuroinflamm 3(5):e257

48. Jarius $\mathrm{S}$ et al (2016) Screening for MOG-IgG and 27 other antiglial and anti-neuronal autoantibodies in 'pattern II multiple sclerosis' and brain biopsy findings in a MOG-IgG-positive case. Mult Scler J 22(12):1541-1549 